\title{
Next-to-next-to-leading order contributions to jet photoproduction and determination of $\alpha_{s}$
}

\author{
Michael Klasen, ${ }^{1, *}$ Gustav Kramer, ${ }^{2}$ and Markus Michael ${ }^{1}$ \\ ${ }^{1}$ Institut für Theoretische Physik, Westfälische Wilhelms-Universität Münster, \\ Wilhelm-Klemm-Straße 9, D-48149 Münster, Germany \\ ${ }^{2}$ II. Institut für Theoretische Physik, Universität Hamburg, \\ Luruper Chaussee 149, D-22761 Hamburg, Germany
}

(Received 7 October 2013; revised manuscript received 27 November 2013; published 18 April 2014)

\begin{abstract}
We present the first calculation of inclusive jet photoproduction with approximate next-to-next-toleading-order contributions, obtained from a unified threshold resummation formalism. The leading coefficients for direct photoproduction are computed analytically. Together with the coefficients pertinent to parton-parton scattering, they are shown to agree with those appearing in our full next-to-leading-order calculations. For hadron-hadron scattering, numerical agreement is found with a previous calculation of jet production at the Tevatron. We show that the direct and resolved approximate next-to-next-to-leading-order contributions considerably improve the description of final ZEUS data on jet photoproduction and that the error on the determination of the strong coupling constant is significantly reduced.
\end{abstract}

DOI: 10.1103/PhysRevD.89.074032

PACS numbers: 12.38.Bx, 13.60.-r

\section{INTRODUCTION}

The HERA collider, which operated at DESY from 1992 to 2007 , has produced many important physics results, first of all perhaps the most precise determinations to date of the quark and gluon densities in the proton from single experiments (H1, ZEUS) [1,2] and their combined data sets [3]. These data, taken in deep-inelastic electron-proton scattering, are complemented by a wealth of data from photoproduction at low virtuality of the exchanged photon, in particular on jet production, giving access also to the distributions of partons in the photon and to measurements of the strong coupling constant [4].

Using the full data set of the HERA run from 2005 to 2007 with an integrated luminosity of $300 \mathrm{pb}^{-1}$, the ZEUS collaboration has recently published a final measurement of inclusive jet photoproduction [5] and used it to determine the strong coupling constant (at the mass $M_{Z}$ of the $Z$ boson) to be

$$
\alpha_{s}\left(M_{Z}\right)=0.1206_{-0.0022}^{+0.0023}(\exp )_{-0.0035}^{+0.0042}(\mathrm{th}),
$$

based on a comparison with our next-to-leading-order (NLO) QCD calculations [6]. While this value (like the one obtained from deep-inelastic electron-photon scattering [7]) is in agreement with the current world average of $\alpha_{s}\left(M_{Z}\right)=0.1184 \pm 0.0007$ [8], Eq. (1) is also less precise, since the latter uses only observables that are known to next-to-next-to-leading order (NNLO) of perturbative QCD.

In this paper, we compute the inclusive jet photoproduction cross section for the first time including

*michael.klasen@uni-muenster.de approximate NNLO (aNNLO) contributions, obtained from a unified threshold resummation formalism [9], and extract the first aNNLO value for the strong coupling constant from photoproduction data. Our calculations are based on our previous work on inclusive jet [10] and dijet [6] photoproduction and lead, as we will see, to a considerably improved description of the ZEUS data and a theoretical error on $\alpha_{s}$ that is significantly reduced.

\section{NNLO CONTRIBUTIONS TO JET PHOTOPRODUCTION}

The QCD factorization theorem allows one to write the differential cross section for inclusive jet photoproduction,

$$
\begin{aligned}
\mathrm{d} \sigma= & \sum_{a, b} \int \mathrm{d} y f_{\gamma / e}(y) \int \mathrm{d} x_{\gamma} f_{a / \gamma}\left(x_{\gamma}, \mu_{\gamma}\right) \int \mathrm{d} x_{p} \\
& \times f_{b / p}\left(x_{p}, \mu_{p}\right) \mathrm{d} \sigma_{a b}\left(\alpha_{s}, \mu ; y, x_{\gamma}, \mu_{\gamma}, x_{p}, \mu_{p}\right),
\end{aligned}
$$

as a convolution of the partonic scattering cross section $\mathrm{d} \sigma_{a b}$, which includes both direct $(a=\gamma, b=q, g)$ and resolved $(a, b=q, g)$ photon contributions, with the Weizsäcker-Williams flux of photons in electrons $f_{\gamma / e}$ and the parton densities in the photon and proton $f_{a / \gamma}$ $\left[\delta\left(1-x_{\gamma}\right)\right.$ for direct photons] and $f_{b / p}$, respectively. Here, $y, x_{\gamma}$, and $x_{p}$ denote the longitudinal momentum fractions of the photon in the electron and of the partons in the photon and proton, respectively, and $\mu, \mu_{\gamma}$, and $\mu_{p}$ are the renormalization and factorization scales.

From a unified threshold resummation formalism, a master formula can be obtained that allows one to compute soft and virtual corrections to arbitrary partonic hard scattering cross sections [9]. At NLO it reads 


$$
\begin{aligned}
\mathrm{d} \sigma_{a b}= & \mathrm{d} \sigma_{a b}^{B} \frac{\alpha_{s}(\mu)}{\pi}\left[c_{3} D_{1}(z)+c_{2} D_{0}(z)+c_{1} \delta(1-z)\right] \\
& +\frac{\alpha_{s}^{d_{\alpha_{s}}+1}(\mu)}{\pi}\left[A^{c} D_{0}(z)+T_{1}^{c} \delta(1-z)\right],
\end{aligned}
$$

where the second line is only present for processes with complex color flow (here resolved processes), $d_{\alpha_{s}}$ denotes the power of $\alpha_{s}$ already present in the Born term $\sigma_{a b}^{B}$ ( 1 for direct and 2 for resolved photoproduction), and

$$
D_{l}(z)=\left[\frac{\ln ^{l}(1-z)}{1-z}\right]_{+}
$$

with decreasing $l$ are the leading and subleading logarithms at partonic threshold $(z \rightarrow 1)$ in pair-invariant-mass kinematics. The NNLO master formula is given in the reference cited above, as are the general and complex color flow formulas for the coefficients $c_{i}, A^{c}$, and $T_{1}^{c}$.

The coefficients for the simple color flow in direct photoproduction are given in the following. For the QCD Compton process $\gamma q \rightarrow q g$, we find $c_{3}=C_{F}-N_{C}$,

$c_{2}=C_{F}\left[-\ln \left(\frac{\mu_{p}^{2}}{s}\right)-\frac{3}{4}+2 \ln \left(\frac{-u}{s}\right)\right]+N_{C} \ln \left(\frac{t}{u}\right)-\frac{\beta_{0}}{4}$,

and the scale-dependent part of the coefficient,

$$
c_{1}^{\mu}=-\frac{3 C_{F}}{4} \ln \left(\frac{\mu_{p}^{2}}{s}\right)+\frac{\beta_{0}}{4} \ln \left(\frac{\mu^{2}}{s}\right) .
$$

For the crossed process $\gamma g \rightarrow q \bar{q}$, we find $c_{3}=2\left(N_{C}-C_{F}\right)$,

$$
c_{2}=-\frac{3 C_{F}}{2}+N_{C}\left[-\ln \left(\frac{\mu_{p}^{2}}{s}\right)+\ln \left(\frac{t u}{s^{2}}\right)\right],
$$

and the scale-dependent part of the coefficient,

$$
c_{1}^{\mu}=-\frac{\beta_{0}}{4} \ln \left(\frac{\mu_{p}^{2}}{s}\right)+\frac{\beta_{0}}{4} \ln \left(\frac{\mu^{2}}{s}\right) .
$$

For both processes, the explicit results for the gaugeindependent parts of the soft anomalous dimension matrix $\Gamma_{S}$ in prompt photon production have been used [9]. The scale-independent parts of $c_{1}$ can be found in Ref. [6]. These coefficients depend on the QCD color factors $C_{F}=$ $4 / 3$ and $N_{C}=3$; the one-loop $\beta$ function $\beta_{0}=\left(11 N_{C}-\right.$ $\left.2 n_{f}\right) / 3$ with $n_{f}$ quark flavors; the Mandelstam variables $s=\left(p_{a}+p_{b}\right)^{2}, \quad t=\left(p_{a}-p_{\text {jet }}\right)^{2}$, and $u=\left(p_{b}-p_{\text {jet }}\right)^{2}$, where $p_{i}$ are the 4-momenta of the participating partons and which satisfy $s+t+u \equiv(1-z) E_{T}^{2} \rightarrow 0$ at partonic threshold; and the renormalization and proton factorization scales $\mu$ and $\mu_{p}$, but not on the photon factorization scale $\mu_{\gamma}$, as the QED splitting of a photon to a quark-antiquark pair is not enhanced by the logarithms given above. We therefore only expect the dependencies on the former two scales to be improved at aNNLO. We have verified that at NLO the direct and resolved coefficients agree with the virtual and soft initial-state corrections calculated in pair-invariantmass kinematics for two jets integrated analytically over singular and numerically over regular regions of phase space [6]. At aNNLO, these coefficients appear exclusively in the three leading logarithmic terms $D_{3}, D_{2}$, and $D_{1}$ of the master formula. For lower terms, only the scale-depenent terms are known and included, but the resulting error should be small.

\section{COMPARISONS WITH DO, ZEUS, AND H1 DATA}

The analytical results described above have been implemented in our program for dijet photoproduction, where a convolution over $z$ was already performed for initial-state singularities [6]. The aNNLO terms for simple color flow were implemented exclusively on the proton side, while those for complex color flow (resolved photoproduction) have been split evenly among the photon and the proton. At NLO, we use of course our complete calculation and not only the logarithmically enhanced terms described above. As a numerical check, we have repeated the calculation of inclusive jet production in $p \bar{p}$ collisions with $\sqrt{s_{p \bar{p}}}=$ $1.8 \mathrm{TeV}$ at the Tevatron at NLO and aNNLO with different scales as shown in Fig. 2 of Ref. [11] (note that the rapidity range there should read $|\eta| \leq 0.5$ ), finding full agreement (see our Fig. 1). Threshold corrections for inclusive jet

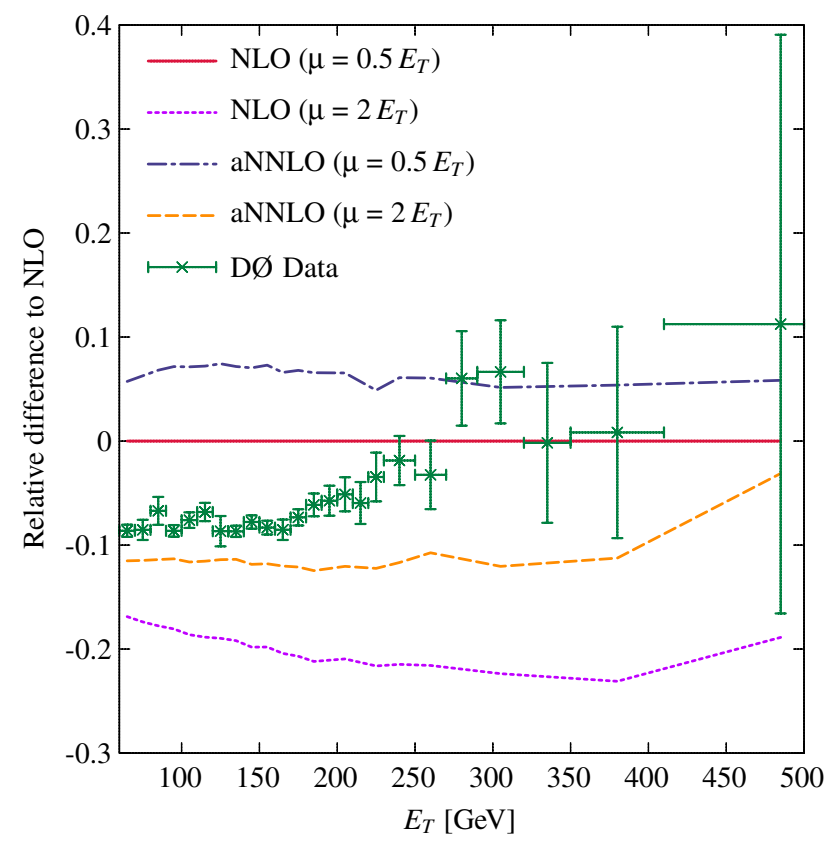

FIG. 1 (color online). Relative difference of D0 data, NLO, and aNNLO predictions with scales $\mu, \mu_{p}=[0.5 ; 2] \times E_{T}$ to the NLO prediction with scales $\mu, \mu_{p}=0.5 \times E_{T}$ as a function of jet transverse energy $E_{T}$. 
hadroproduction have also been studied in the past years by other authors [12,13]. At the central rapidities and high transverse energies $E_{T}$ measured, the partonic momentum fractions $x_{p, \bar{p}}=2 E_{T} / \sqrt{s_{p \bar{p}}} \rightarrow 1 / 1.8$ can be seen to be sufficiently close to 1 for these corrections to be relevant.

The final ZEUS measurements for inclusive jet photoproduction and the determination of $\alpha_{s}$ were performed with photon virtuality $Q^{2}<1 \mathrm{GeV}^{2}, \gamma p$ center-of-mass energy in the range $142<W_{\gamma p}<293 \mathrm{GeV}$, and using the inclusive $k_{T}$ algorithm [14] with jet radius $R=1$, transverse energy $E_{T}>17 \mathrm{GeV}$, and rapidity in the range $-1<\eta<2.5$ [5]. As for the Tevatron, the partonic momentum fractions $x_{\gamma, p}=2 E_{T} / \sqrt{W_{\gamma p}} \rightarrow 70 / 142$ are sufficiently close to 1 for the threshold corrections to be relevant. To facilitate easy comparison of the following figures, we also employ the ZEUS-S [15] and GRV-HO [16] fits of the parton densities in the proton and photon, where the latter has been transformed from the DIS $\gamma$ to the $\overline{\mathrm{MS}}$ scheme. Similar measurements were also performed almost a decade earlier by the H1 collaboration [17]. Because of the lower integrated luminosity available then $\left(24.1 \mathrm{pb}^{-1}\right)$, the data are less precise, but we have verified that within errors they are in good agreement with our calculations at NLO and aNNLO.

Focusing now on the more precise ZEUS data, we compare in Fig. 2 the measured and various theoretical transverse energy spectra to the NLO prediction with central scales $\left(\mu, \mu_{\gamma}, \mu_{p}=E_{T}\right)$ and after applying hadronization corrections [5]. The NLO uncertainty band (blue, color online) is obtained by varying the scales about the

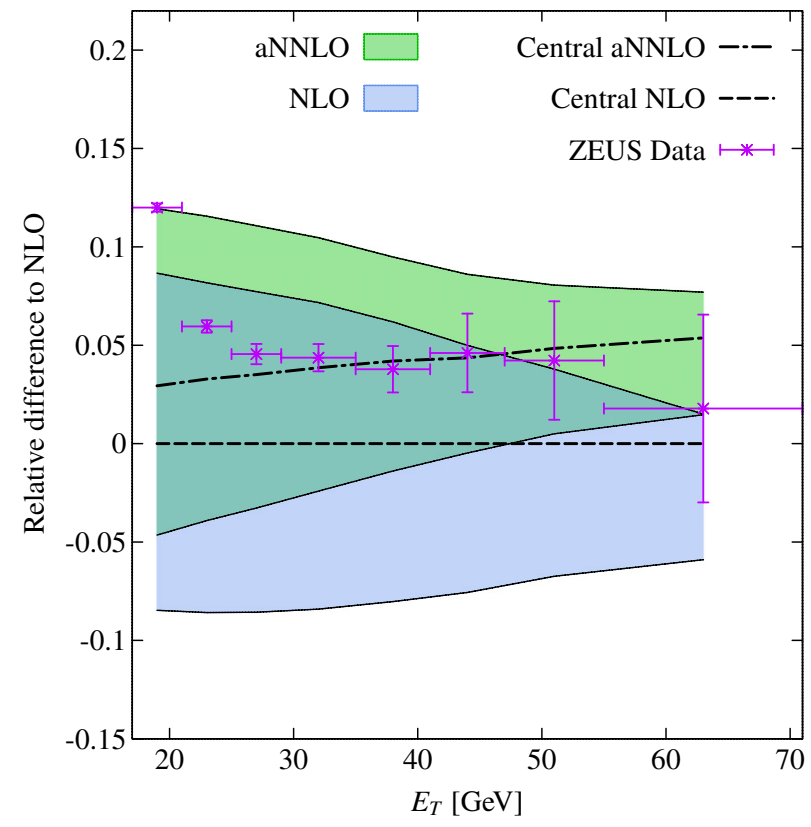

FIG. 2 (color online). Relative difference of ZEUS data, NLO, and aNNLO predictions with scales $\mu, \mu_{\gamma}, \mu_{p}=[0.5 ; 2] \times E_{T}$ to the central NLO prediction with scales $\mu, \mu_{\gamma}, \mu_{p}=E_{T}$ as a function of jet transverse energy $E_{T}$. central scale up and down by a factor of 2 and coincides with the one shown in Fig. 2 of Ref. [5]. The aNNLO corrections (including NNLO running of $\alpha_{s}$ ) increase the central prediction by $3-6 \%$, bringing it into considerably better agreement with the experimental data. As expected from the general behavior of threshold logarithms, the increase is larger at high $E_{T}$. The scale uncertainty is also reduced at aNNLO (green band), in particular at high $E_{T}$, where it drops from $8 \%$ to $5 \%$. Note that the data point in the largest $E_{T}$ bin has been omitted from this comparison, as it suffers from large experimental (in particular jet energy scale) uncertainties.

In Fig. 3 we perform a similar comparison for the jet rapidity distribution. This distribution has been problematic since the earliest HERA runs, as it tended to be overestimated in the backward (photon) and underestimated in the forward (proton) direction. These discrepancies are indeed observed at NLO in Fig. 3, together with a large scale uncertainty, in particular in the forward direction. They were traditionally assigned to hadronization but also missing higher-order corrections. This conjecture can now be corroborated for the first time, as the aNNLO contributions do bring the theoretical predictions into better agreement with the data in both kinematical regions. The scale uncertainty is not significantly improved at aNNLO, as the data are dominated by the low- $E_{T}$ region, but within it they can now be described up to the largest rapidities. Note that all figures in this section have been obtained using the world average value for $\alpha_{s}\left(M_{Z}\right)=0.118$ as required for the ZEUS-S (standard) fit [15].

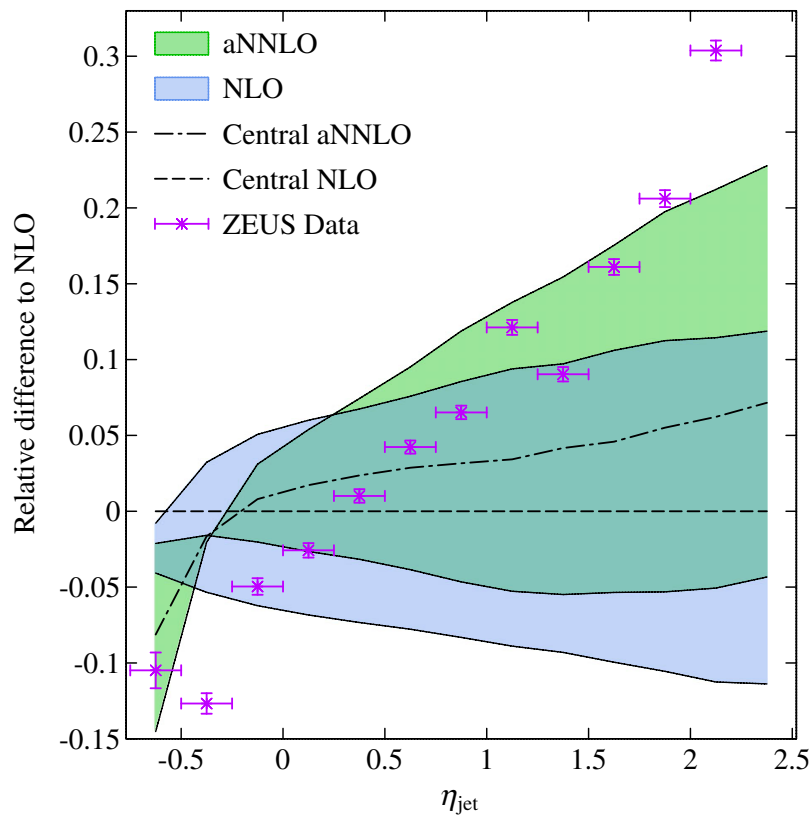

FIG. 3 (color online). Relative difference of ZEUS data, NLO, and aNNLO predictions with scales $\mu, \mu_{\gamma}, \mu_{p}=[0.5 ; 2] \times E_{T}$ to the central NLO prediction with scales $\mu, \mu_{\gamma}, \mu_{p}=E_{T}$ as a function of jet rapidity $\eta$ for $E_{T}>17 \mathrm{GeV}$. 


\section{DETERMINATION OF $\boldsymbol{\alpha}_{s}$}

To determine the strong coupling constant from these comparisons, the theoretical calculations have to be performed with a set of parton densities in the proton obtained from global fits assuming different values of $\alpha_{s}\left(M_{Z}\right)$. For our analysis at aNNLO, we employ the latest fits of the CTEQ-TEA collaboration (CT10), which have been obtained with a NNLO running of the coupling, evolution of the parton densities, deep-inelastic scattering, and vector-boson production matrix elements [18]. 13 different CT10 NNLO sets were used, which correspond to values of $\alpha_{s}\left(M_{Z}\right)=0.112$ to 0.124 . In contrast, the ZEUS determination at NLO in Eq. (1) was based on only five different sets of ZEUS-S parton densities corresponding to values of $\alpha_{s}\left(M_{Z}\right)=0.115$ to 0.123 . Its theoretical error was dominated by higher-order terms, estimated from scale variations, but depended only weakly on variations of the parton densities in the proton, the hadronization model, and the jet algorithm.

At NLO, for a scale choice of $\mu, \mu_{\gamma}, \mu_{p}=E_{T}$, and omitting the lowest $E_{T}$ point, which lies clearly outside the theoretical error band at this order (see Fig. 2), we reproduce $\alpha_{s}\left(M_{Z}\right)=0.121_{-0.002}^{+0.002}(\exp )_{-0.003}^{+0.005}(\mathrm{th})$ as in Eq. (1) and the ZEUS analysis [5]. As stated there, including the lowest $E_{T}$ point severely worsens the quality of the fit, and we find an increase in the minimal value of $\chi^{2} /$ d.o.f. from $16 / 6$ to $123 / 7$. Through this result, we also confirm that the fit of the strong coupling constant does not depend very much on the employed parton densities in the proton, which were CT10 NNLO in our fit and ZEUS-S NLO in the ZEUS analysis. The uncertainty induced by the parton densities in the photon was systematically studied by the ZEUS collaboration and estimated to be $+2 /-1 \%$. It is expected to remain the same at NLO and aNNLO, in particular due to the lack of more precise deep-inelastic electron-photon scattering data and a NNLO fit to them.

Finally, at aNNLO and for the same scale choice as the one given above, we obtain

$$
\alpha_{s}\left(M_{Z}\right)=0.120_{-0.002}^{+0.002}(\exp )_{-0.003}^{+0.003}(\mathrm{th}) \text {. }
$$

The central value is now lower, since the aNNLO contributions increase the cross section for all $E_{T}$ bins (see Fig. 2) and is brought closer to the world average of 0.118 . In addition, the theoretical error is significantly reduced, which reflects the stabilization of the cross section prediction with respect to variations of the unphysical scales.

\section{CONCLUSIONS}

In conclusion, we have presented here a first calculation of inclusive jet photoproduction up to aNNLO of perturbative QCD. Leading and subleading logarithmic contributions were extracted from a unified threshold resummation formalism for photon-parton and parton-parton scattering processes pertinent to direct and resolved photoproduction of jets and shown to agree with those appearing in our full NLO calculations. The aNNLO contributions implemented in our NLO program were shown to correctly reproduce results obtained in the literature for hadronhadron scattering at the Tevatron and to considerably improve the description of final ZEUS data on jet photoproduction. An aNNLO fit of these data with the CT10 set of parton densities resulted in a new determination of the strong coupling constant at the mass of the $Z$ boson in agreement with the current world average and the ZEUS determination at NLO, but with a significantly reduced theoretical error.

\section{ACKNOWLEDGMENTS}

We thank C. Glasman, N. Kidonakis, and W. Vogelsang for useful discussions. This work has been supported by the BMBF Theorie-Verbund "Begleitende theoretische Untersuchungen zu den Experimenten an den Großgeräten der Teilchenphysik."
[1] C. Adloff et al. (H1 Collaboration), Eur. Phys. J. C 21, 33 (2001).

[2] S. Chekanov et al. (ZEUS Collaboration), Eur. Phys. J. C 21, 443 (2001).

[3] F. D. Aaron et al. (H1 and ZEUS Collaboration), J. High Energy Phys. 01 (2010) 109.

[4] M. Klasen, Rev. Mod. Phys. 74, 1221 (2002).

[5] H. Abramowicz et al. (ZEUS Collaboration), Nucl. Phys. B864, 1 (2012).

[6] M. Klasen and G. Kramer, Z. Phys. C 72, 107 (1996); 76, 67 (1997); M. Klasen, T. Kleinwort, and G. Kramer, Eur. Phys. J. direct C 1, 1 (1998).
[7] S. Albino, M. Klasen, and S. Söldner-Rembold, Phys. Rev. Lett. 89, 122004 (2002).

[8] J. Beringer et al. (Particle Data Group Collaboration), Phys. Rev. D 86, 010001 (2012).

[9] N. Kidonakis, Int. J. Mod. Phys. A 19, 1793 (2004).

[10] M. Klasen, G. Kramer, and S. G. Salesch, Z. Phys. C 68, 113 (1995).

[11] N. Kidonakis and J. F. Owens, Phys. Rev. D 63, 054019 (2001).

[12] D. de Florian and W. Vogelsang, Phys. Rev. D 76, 074031 (2007); A. Mukherjee and W. Vogelsang, Phys. Rev. D 86, 094009 (2012). 
[13] M. C. Kumar and S. O. Moch, Phys. Lett. B 730, 122 (2014).

[14] S. Catani, Y. L. Dokshitzer, M. H. Seymour, and B. R. Webber, Nucl. Phys. B406, 187 (1993); S. D. Ellis and D. E. Soper, Phys. Rev. D 48, 3160 (1993).

[15] S. Chekanov et al. (ZEUS Collaboration), Phys. Rev. D 67, 012007 (2003).
[16] M. Glück, E. Reya, and A. Vogt, Phys. Rev. D 45, 3986 (1992); 46, 1973 (1992).

[17] C. Adloff et al. (H1 Collaboration), Eur. Phys. J. C 29, 497 (2003).

[18] J. Gao, M. Guzzi, J. Huston, H.-L. Lai, Z. Li, P. Nadolsky, J. Pumplin, D. Stump, and C.-P. Yuan, Phys. Rev. D 89, 033009 (2014). 